\title{
Triage Notes in Syndromic Surveillance - A Double Edged Sword
}

\author{
Zachary Faigen ${ }^{\star 1}$, Amy Ising $^{2}$, Lana Deyneka ${ }^{1}$ and Anna E. Waller ${ }^{2}$ \\ ${ }^{1} \mathrm{NC}$ Department of Health and Human Services, Raleigh, NC, USA; ${ }^{2}$ North Carolina Center for Health Informatics, Chapel Hill, NC, \\ USA
}

\section{Objective}

This roundtable will provide a forum for the ISDS community to discuss the use of emergency department (ED) triage notes in syndromic surveillance. It will be an opportunity to discuss both the benefits of having this variable included in syndromic surveillance data feeds, as well as the drawbacks and challenges associated with working with such a detailed data field.

\section{Introduction}

The advent of Meaningful Use (MU) has allowed for the expansion of data collected at the hospital level and received by public health for syndromic surveillance. The triage note, a free text expansion on the chief complaint, is one of the many variables that are becoming commonplace in syndromic surveillance data feeds. Triage notes are readily available in many ED information systems, including, but not limited to, Allscripts, Cerner, EPIC, HMS, MedHost, Meditech, and T-System. North Carolina's syndromic surveillance system, NC DETECT, currently collects triage notes from 33 out of 122 hospitals in the State $(27 \%)$, and this number is likely to increase.

\section{Description}

Representatives from the Carolina Center for Health Informatics (CCHI) in the University of North Carolina Department of Emergency Medicine, and the North Carolina Division of Public Health (DPH) will describe their experiences with triage notes to date and engage the audience in the discussion. There are unique rewards and challenges for both groups. Presenting these issues in an organized manner will allow participants to be part of a robust conversation that covers numerous topics, from informatics to public health surveillance practice.

The CCHI team will describe current approaches to triage note processing, including the use of open source tools the Emergency Medical Text Processor (EMT-P) and the negation tool NegEx. Pros and cons of the current approach will be presented with attendee discussion of potential alternative approaches.

The epidemiologists at DPH, who review the syndromic surveillance data on a daily basis, benefit greatly from the addition of triage notes. They provide a wealth of information that can lead to the identification of disease clusters, outbreaks, cases related to ongoing outbreaks, or other events of public health importance that would otherwise not be detected. They can also aid in the follow-up and investigation process. However, this plethora of text can also cause many issues for the reviewer, such as increasing the amount of time it takes to review records and the alteration in the behavior of established signal/alert and report processes.

\section{Audience Engagement}

Experiences and activities regarding the use of triage notes in North Carolina will be presented to the audience from both the data processor and data reviewer perspectives. Examples of triage notes and their application in surveillance practice will be provided. Current status, successes, and lessons learned will be identified. An open discussion will be encouraged so that the audience will have an opportunity to share their own experiences, practices, and questions regarding the use of triage notes in syndromic surveillance. The outcome of the roundtable will be the identification of best practices and the potential creation of a triage note working group to build upon the current successes that North Carolina and other jurisdictions have achieved.

Example facilitation questions include the following:

What are the pros and cons of collecting triage notes?

How do you handle negation in triage notes?

How did you handle the documentation of Ebola screens that often appear in triage notes?

\section{Keywords}

Informatics; Syndromic Surveillance; Triage Notes; Emergency Department Data

\section{*Zachary Faigen}

E-mail: zachary.faigen@dhhs.nc.gov 